Pacific Journal of Mathematics

A UNIFIED APPROACH TO BOUNDARY VALUE PROBLEMS 


\title{
A UNIFIED APPROACH TO BOUNDARY VALUE PROBLEMS ON COMPACT INTERVALS
}

\author{
FrankLin T. IHA
}

\begin{abstract}
Let $L$ be a formal differential operator of order $n$ and consider $L$ as an operator from $C^{n}([a, b]) \subset L^{2}([a, b])$ into $L^{2}([a, b])$. Let $\left\{\eta_{1}, \cdots, \eta_{l}\right\}$ be a set of linear functionals defined on $C^{n}([a, b])$ with the property that each $\eta_{j} T, j=$ $1, \cdots, l$, is continuous, where $T$ is a continuous right inverse of $L$. Let $M$ be the set of all $f \in C^{n}([a, b])$ such that $\eta_{j}(f)=0$, $1 \leqq j \leqq l$, and $N$ be the set of all $f \in M$ such that $L f=0$. It is shown that the inverse of $L$ from $L(M)$, the image of $M$ under $L$, into $M \cap N^{\perp}$ is a compact operator and can be represented as an integral operator. In particular, if $l=n$ and $\left\{\eta_{j}\right\}$ is linearly independent, the inverse of $L$ maps $C([a, b])$ onto $M$ and it is compact. The Hilbert-Schmidt expansion theorem is generalized to these inverse operators when $L$ is self-adjoint on $M$.
\end{abstract}

The purpose of this paper is to put the homogeneous boundary value problems of ordinary differential equations in an abstract setting, so that the properties which make the whole analysis go through become transparent. We replace the usual boundary condition with a linear functional $\eta$ defined on $C^{n}([a, b])$, where $n$ is the order of the differential equation. This has been done by many authors, but their main purpose was to facilitate the notation and, with the exception of Calkin in [1] and Dunford and Schwartz in [2], the topological property of $\eta$ was never used. In this paper, however, the continuity of $\eta T$, where $T$ is an operator whose construction will be given later, is essential. It is this property which will make the integral representation of the inverse of a differential operator possible. Once the integral representation is obtained, we can generalize the Hilbert-Schmidt expansion theorem to this inverse operator when the differential operator is self-adjoint on $M$ where $M$ is the set of all functions in $C^{n}([a, b])$ satisfying the boundary conditions. The integral representation of the inverse of a differential operator has been obtained almost always through the use of the Green's function associated with the boundary value problem. But it is easy to find an example for which the Green's function does not exist. Even in such a case, however, we can construct an inverse of a differential operator $L$ from the range $L(M)$ of $L$ on $M$ into $M \cap N^{\perp}$, where $N$ is the null space of $L$ in $M$, and this is the operator for which we obtain an integral representation and prove that it is compact. 
2. Notations. Throughout the paper $L$ denotes the operator defined by

$$
(L f)(x)=\sum_{k=0}^{n} P_{k}(x) f^{(n-k)}(x), \quad f \in C^{n}([a, b]),
$$

where each $P_{k}(x)$ is a continuous function on the closed finite interval $[a, b]$ and $P_{0}(x) \neq 0$ for any $x \in[a, b]$. We regard $L$ as a mapping from $C^{n}([a, b]) \subset L^{2}([a, b])$ into $C([a, b]) \subset L^{2}([a, b])$, and whenever continuity is mentioned, it is with respect to the norm of $L^{2}([a, b])$. All the functions are restrictions to $[a, b]$. Thus we simply write $L f=0$ meaning $(L f)(x)=0$ for all $x \in[a, b]$.

We set

$$
S=\left\{f \in C^{n}([a, b]) \mid L f=0\right\} .
$$

It is a classical result that $S$ is an $n$-dimensional linear space.

3. Generalized homogeneous boundary value problems. We first of all construct an operator $T$ from $C([a, b])$ into $C^{n}([a, b])$ having the property $L T f=f$ for all $f \in C([a, b])$. For this purpose we take a basis $\left\{y_{j}(x)\right\}$ of $S$ and consider the system of equations

$$
\sum_{k=1}^{n} a_{k}(x) y_{k}^{(j-1)}(x)=\delta_{j n} f(x) / P(x), \quad 1 \leqq j \leqq n,
$$

where $\delta_{j_{n}}=0$ if $j \neq n$ and $\delta_{n n}=1$. Since $\left\{y_{j}\right\}$ is a basis for $S$ and $P_{0}(x) \neq 0$ for any $x \in[a, b]$, the Wronskian $W(x)$ of $\left\{y_{j}\right\}$ does not vanish at any point of $[a, b]$. Hence we can solve the system (3) for $a_{k}(x)$ and obtain

$$
a_{j}(x)=F_{j}(x) f(x), \quad 1 \leqq j \leqq n,
$$

in which each $F_{j}$ is of the form $Q_{j}(x)\left[P_{0}(x) W(x)\right]^{-1}$ where $Q_{j}(x)$ is a polynomial in $y_{j}^{(k-1)}(x), 1 \leqq j \leqq n, 1 \leqq k \leqq n$. Since $W(x) \neq 0$ for any $x \in[a, b]$, it follows each $F_{j}$ is continuous on $[a, b]$. We define $T$ by

$$
(T f)(x)=\int_{a}^{x}\left[\sum_{j=1}^{n} y_{j}(x) F_{j}(\xi)\right] f(\xi) d \xi, \quad f \in C[a, b] .
$$

From (3) and (4) we obtain

$$
\sum_{k=1}^{n} y_{k}^{(j-1)}(x) F_{k}(x)=\delta_{j n} / P_{0}(x), \quad 1 \leqq j \leqq n .
$$

From (5) and (6) we obtain the following properties of $T$ : For each $f \in C([a, b])$,

$$
T f \in C^{n}([a, b])
$$




$$
\begin{aligned}
& D^{k}(T f)(x)=\int_{a}^{x}\left[\sum_{j=1}^{n^{\prime}} y_{j}^{(k)}(x) F_{j}(\xi)\right] f(\xi) d \xi \\
& D^{n}(T f)(x)=f(x)+\int_{a}^{x}\left[\sum_{j=1}^{n} y_{j}^{(n)}(x) F_{j}(\xi)\right] f(\xi) d \xi
\end{aligned}
$$

$$
L T f=f .
$$

The clue to the generalization to the standard boundary value problems is given by the following very simple observation:

LEMma 1. Let $T$ be the operator defined by (5) and $c \in[a, b]$. Let $\zeta_{k, c}$ be the linear functional defined by $\zeta_{k, c}(f)=f^{(k)}(x), f \in C^{n}([a, b])$, $0 \leqq k \leqq n-1$. Then each $\zeta_{k, c} T, 0 \leqq k \leqq n-1$, is continuous, that $i s$, there exists a constant $A_{k}$ such that

$$
\left|\zeta_{k, c}(T f)\right| \leqq A_{k}\|f\|, \quad f \in C([a, b]),
$$

where $\|$ II denotes the norm of $L^{2}([a, b])$.

From the lemma it follows that the linear functional $\eta$ occurring in the usual boundary value problems all have the property that $\eta T$ is continuous.

LEMMA 2. Let $T_{0}$ be an operator having the following properties:

(i) $L T_{0} f=f$ for all $f \in C([a, b])$,

(ii) $\left\|T_{0} f\right\| \leqq A\|f\|$ for all $f \in C([a, b])$.

If $\eta$ is a linear functional such that $\eta T_{0}$ is continuous, then $\eta T$ is also continuous.

Proof. Let $\left\{z_{1}, z_{2}, \cdots, z_{n}\right\}$ be an orthonormal basis for $S$. Then $T f-T_{0} f \in S$ and in fact $T f-T_{0} f=\sum_{j=1}^{n}\left(T f-T_{0} f, z_{j}\right) z_{j}$. Hence,

$$
\eta T f=\eta T_{0} f+\sum_{j=1}^{n}\left(T f-T_{0} f, z_{j}\right) \eta\left(z_{j}\right)
$$

from which the assertion follows.

Definition 1. Let $T$ be the operator defined by (5). A linear functional $\eta$ defined on $C^{n}([a, b])$ is said to be a boundary functional for $L$ if it has the property that $\eta T$ is continuous (with respect to the norm of $\left.L^{2}([a, b])\right)$.

Definition 2. A set $\left\{\eta_{1}, \cdots, \eta_{l}\right\}$ of boundary functionals for $L$ is said to be linearly independent if they are linearly independent as duals on $S$, that is, if $\sum_{j=1}^{l} \alpha_{j} \eta_{j}(f)=0$ for all $f \in S$ implies $\alpha_{j}=0$, $1 \leqq j \leqq l$, where $S$ is the set defined in (2), or equivalently, if for 
any basis $\left\{f_{j}\right\}$ of $S$, the rank of the matrix $\left[\eta_{i}\left(f_{j}\right)\right]$ is $l$.

As a direct generalization of the usual homogeneous boundary value problem, we have

THEOREM 1. Let $\left\{\eta_{1}, \cdots, \eta_{n}\right\}$ be a set of linearly independent boundary functionals for $L$ and let

$$
M=\left\{f \in C^{n}([a, b]) \mid \eta_{j}(f)=0, \quad 1 \leqq j \leqq n\right\} .
$$

Then the inverse $K$ of $L$ from $C([a, b])$ into $M$ exists, and it is compact. Moreover, there exists a function $K(x, \xi)$ having the following properties:

$$
(K f)(x)=\int_{a}^{b} K(x, \xi) f(\xi) d \xi, \quad f \in C([a, b]) ;
$$

for each $x \in[a, b]$

$$
K(x, \xi) \in L^{2}([a, b])
$$

and

$$
\int_{a}^{b}|K(x, \xi)|^{2} d \xi \leqq B^{2} \text { for all } x \in[a, b]
$$

for some constant $B$.

Proof. Let $\left\{y_{1}, \cdots, y_{n}\right\}$ be a basis for $S$. It is straightforward to show that $L$ is one-to-one on $M$.

Since $\operatorname{det}\left[\eta_{i}\left(y_{j}\right)\right] \neq 0$, given $f \in C([a, b])$, there exist unique $C_{\jmath}$, $1 \leqq j \leqq n$, such that

$$
\sum_{j=1}^{n} \eta_{2}\left(y_{j}\right) C_{j}=-\eta_{2}(T f), \quad 1 \leqq i \leqq n
$$

Moreover, each $C_{j}$ is of the form

$$
C_{j}=\sum_{k=1}^{n} \alpha_{j k} \eta_{k}(T f)
$$

where $\alpha_{k j}$ are constants which depend only on $\eta_{\imath}\left(y_{j}\right), 1 \leqq i \leqq n$, $1 \leqq j \leqq n$. From (14) and (15) we obtain

$$
\eta_{i}\left(\sum_{j=1}^{n} \sum_{k=1}^{n} \alpha_{j k} \eta_{k}(T f) y_{j}+T f\right)=0, \quad 1 \leqq i \leqq n .
$$

We set

$$
\left(T_{0} f\right)(x)=\sum_{j=1}^{n} \sum_{k=1}^{n} \alpha_{j k} \eta_{k}(T f) y_{j}(x), \quad f \in C([a, b])
$$

and 


$$
K f=T_{0} f+T f, \quad f \in C([a, b]) .
$$

From (16), (17), and (18) we have $\eta_{i}(K f)=0,1 \leqq i \leqq n, f \in C([a, b])$, so that $K f \in M$ for all $f \in C([a, b])$. Moreover, from (9), (17), and (18), we see that $L(K f)=f$ for all $f \in C([a, b])$. The compactness of $K$ follows from the fact that $T_{0}$ is a continuous operator with finite dimensional range, so that $T_{0}$ (along with $T$ ) is also compact. From the hypothesis, for each $j, 1 \leqq j \leqq n$, there exists a constant $A_{j}$ such that

$$
\left|\eta_{j}(T f)\right| \leqq A_{j}\|f\|, \quad f \in C([a, b]) .
$$

Since $C([a, b])$ is dense in $L^{2}([a, b])$, we can extend the continuous linear functional $f \rightarrow \eta_{j}(T f)$ continuously to $L^{2}([a, b])$ with the same bound $A_{j}$. Hence it follows that there exists $G_{j} \in L^{2}([a, b])$ such that

$$
\eta_{j}(T f)=\int_{a}^{b} G_{j}(\xi) f(\xi) d \xi, \quad f \in C([a, b])
$$

and

$$
\int_{a}^{b}\left|G_{j}(\xi)\right|^{2} d \xi \leqq A_{j}^{2}
$$

Substituting (19) in (17), we obtain

$$
\left(T_{0} f\right)(x)=\int_{a}^{b}\left[\sum_{i=1}^{n} \sum_{j=1}^{n} \alpha_{i j} y_{i}(x) G_{j}(\xi)\right] f(\xi) d \xi, \quad f \in C([a, b]) .
$$

We set

$$
\begin{gathered}
J_{0}(x, \xi)=\sum_{i=1}^{n} \sum_{j=1}^{n} \alpha_{i j} y_{i}(x) G_{j}(\xi) \\
J(x, \xi)=\sum_{j=1}^{n} y_{j}(x) F_{j}(\xi) \text { if } a \leqq \xi \leqq x \\
=0 \text { if } \xi>x, \\
K(x, \xi)=J_{0}(x, \xi)+J(x, \xi) .
\end{gathered}
$$

Then, from (18), (5), (23), (21), (22), and (24) we obtain

$$
(K f)(x)=\int_{a}^{b} K(x, \xi) f(\xi) d \xi, \quad f \in C([a, b]) .
$$

From (20), (22), (23), and (24) and from the fact that $y_{j}$ and $F_{j}$, $1 \leqq j \leqq n$, are continuous functions on the compact set $[a, b]$, it follows that $K(x, \xi)$ has the desired properties (12) and (13).

We next consider the case in which the number of boundary functionals is not necessarily $n$ and may not be linearly independent. 
THEOREM 2. Let $\left\{\eta_{j}\right\}_{1}^{l}$ be a finite collection of boundary functionals for $L$. Let

$$
\begin{aligned}
M_{0} & =\left\{f \in C^{n}([a, b]) \mid \eta_{j}(f)=0, \quad 1 \leqq j \leqq l\right\} \\
N & =M_{0} \cap S=\left\{f \in M_{0} \mid L f=0\right\} \\
M_{1} & =M_{0} \cap N^{\perp}=\left\{f \in M_{0} \mid(f, g)=0 \text { for all } g \in N\right\} \\
L\left(M_{0}\right) & =\left\{f \mid f=L u \text { for some } u \in M_{0}\right\} .
\end{aligned}
$$

Then there exists an operator $K_{1}$ from $L\left(M_{0}\right)$ into $M_{1}$ such that

(i) $L K_{1} f=f$ for all $f \in L\left(M_{0}\right)$,

(ii) $K_{1}$ is compact on $L\left(M_{0}\right)$,

(iii) there exists a function $K_{1}(x, \xi)$ such that $\left(K_{1} f\right)(x)=$ $\int_{a}^{b} K_{1}(x, \xi) f(\xi) d \xi, f \in L\left(M_{0}\right)$; for each $x \in[a, b], K_{1}(x, \xi) \in L^{2}([a, b])$, and $\int_{a}^{b}\left|K_{1}(x, \xi)\right|^{2} d \xi \leqq B_{1}^{2}$ for all $x \in[a, b]$ for some constant $B_{1}$.

Proof. Let $\operatorname{dim} N=p$. Let $\left\{g_{j}\right\}$ be an orthonormal basis for $N$ and let

$$
\eta_{l+j}(f)=\left(f, g_{j}\right), \quad f \in L^{2}([a, b]), \quad 1 \leqq j \leqq p .
$$

Then clearly $\eta_{l+j}, \quad 1 \leqq j \leqq p$, are boundary functionals for $L$. Moreover,

$$
M_{1}=\left\{f \in C^{n}([a, b]) \mid \eta_{j}(f)=0, \quad 1 \leqq j \leqq l+p\right\} .
$$

Since $M_{0}=M_{1} \oplus N$ and $N$ is the null space of $L$ on $M_{0}, \quad L$ is oneto-one on $M_{1}$, so that if a solution in $M_{1}$ of the equation $L y=f$ does exist, it is unique. Let $\left\{y_{i}\right\}$ be a basis for $S$. Then if $f \in L\left(M_{0}\right)=$ $L\left(M_{1}\right)$, there exist unique $C_{j}, 1 \leqq j \leqq n$, such that

$$
\sum_{j=1}^{n} C_{j} y_{j}(x)+(T f)(x)
$$

is the solution of $L y=f$ in $M_{1}$, that is,

$$
L\left(\sum_{j=1}^{n} C_{j} y_{j}+T f\right)=f
$$

and

$$
\eta_{i}\left(\sum_{j=1}^{n} C_{j} y_{j}+T f\right)=0, \quad 1 \leqq i \leqq l+p .
$$

From (26) it is seen that each $C_{j}$ is of the form

$$
C_{j}=\sum_{k=1}^{l+p} \alpha_{j k} \eta_{k}(T f)
$$

where $\alpha_{j k} \in C$ depend only on $\eta_{j}\left(y_{k}\right), 1 \leqq j \leqq l+p, 1 \leqq k \leqq n$. Let 


$$
\left(K_{1} f\right)(x)=\sum_{i=1}^{n} \sum_{j=1}^{l+p} \alpha_{i j} \eta_{j}(T f) y_{i}(x)+(T f)(x), \quad f \in L\left(M_{0}\right) .
$$

Then from (25), (26), and (27) we have $K_{1} f \in M_{1}$ and $L\left(K_{1} f\right)=f$ for all $f \in L\left(M_{0}\right)$. The proof that $K_{1}$ is compact and that it can be represented as an integral operator satisfying (i)-(iii) is the same as in the proof of Theorem 1 .

4. Generalized Hilbert-Schmidt theorems. We get back to Theorem 1. We need to extend the operator $K$ to $L^{2}([a, b])$ and establish some elementary facts before we can state the next theorem.

Since each $\eta_{j} T$ is a linear functional continuous on the dense set $C([a, b])$, it has a unique continuous extension to $L^{2}([a, b])$. Hence $T_{0}$ has a unique continuous extension $\widehat{T}_{0}$ to $L^{2}([a, b])$. We observe that

$$
\widehat{T}_{0} f \in C^{n}([a, b]) \text { for all } f \in L^{2}([a, b]) \text {. }
$$

The integral defining $T$ makes sense even if $f \in L^{2}([a, b])$, and denoting this extended operator by $\hat{T}$, it is easily seen from (5) that

$$
\widehat{T} f \in C([a, b]) \text { for all } f \in L^{2}([a, b]) \text {. }
$$

Let

$$
\hat{K}=\widehat{T}_{0}+\hat{T} .
$$

Then $\hat{K}$ is the continuous extension of $K$ and $\hat{K}$ is compact on $L^{2}([a, b])$. From $(28)$ and $(29)$, we have

$$
\hat{K} f \in C([a, b]) \text { for all } f \in L^{2}([a, b]) .
$$

With these definitions and notations, we have the following lemma.

Lemma 3. Let $\lambda$ be a nonzero eigenvalue of $\hat{K}$ and $\phi$ be the corresponding eigenfunction. Then $\varphi \in C^{n}([\alpha, b])$, and in fact $\varphi \in M$, where $M$ is defined by (10).

Proof. By definition, $\hat{K} \varphi=\lambda \varphi$ and $\varphi \in L^{2}([a, b])$. Hence from (31) we have $\hat{K}_{\varphi} \in C([a, b])$. Since $\lambda \neq 0$, it follows $\varphi \in C([a, b])$. But this means that $\hat{K}_{\varphi}=K_{\varphi} \in M$, so that $\varphi \in M$.

From this lemma it follows that every eigenfunction of $\hat{K}$ corresponding to a nonzero eigenvalue is also an eigenfunction of $L$.

We now prove the Hilbert-Schmidt theorem for the inverse operator of Theorem 1 when $L$ is self-adjoint on $M$, that is, when $(L f, g)=(f, L g)$ for all $f, g \in M$. (For the conditions as to what 
differential operators can be self-adjoint, see Naimark [3].) Before we state the theorem, however, we observe that if $L$ is self-adjoint on $M, K$ is self-adjoint on $C([a, b])$ and so $\hat{K}$ is self-adjoint on $L^{2}([a, b])$. Moreover, since the range of $\hat{K}$ contains an infinite dimensional subspace $M$, it follows that $\hat{K}$ has infinitely many eigenvalues. With these and the elementary properties of self-adjoint compact operators on a Hilbert space in mind, we state the theorem.

THEOREM 3. Let $L$ have the additional property that it is self-adjoint on $M$. Let $\left\{\varphi_{j}\right\}$ be the complete system of orthonormal eigenfunctions of $\hat{K}$ corresponding to nonzero eigenvalues, and $\lambda_{j}$ be the eigenvalue of $\hat{K}$ corresponding to $\varphi_{j}$. Then for each $f \in L^{2}([a, b])$,

$$
\hat{K} f=\sum_{j=1}^{\infty}\left(\hat{K} f, \varphi_{j}\right) \varphi_{j}=\sum_{j=1}^{\infty} \lambda_{j}\left(f, \varphi_{j}\right) \varphi_{j}
$$

and the series converges to $\hat{K} f$ uniformly on $[a, b]$.

Proof. The fact that the series converges to $\hat{K} f$ in $L^{2}([a, b])$ follows from the theory of self-adjoint compact operators on a Hilbert space.

Using the Cauchy's inequality, we obtain

$$
\left|\sum_{j=1}^{m}\left(f, \varphi_{j}\right) \lambda_{j} \varphi_{j}(x)\right| \leqq\left[\sum_{j=1}^{m}\left|\left(f, \varphi_{j}\right)\right|^{2}\right]^{1 / 2}\left[\sum_{j=1}^{m} \lambda_{j}^{2}\left|\varphi_{j}(x)\right|^{2}\right]^{1 / 2} \text {. }
$$

Since $\lambda_{j} \varphi_{j}(x)=\int_{a}^{b} K(x, \xi) \varphi_{j}(\xi) d \xi$, fixing $x \in[a, b]$, recalling (12) and (13), and applying the Bessel's inequality to the function $K(x, \xi)$, we obtain

$$
\sum_{j=1}^{\infty} \lambda_{j}^{2}\left|\varphi_{j}(x)\right|^{2} \leqq \int_{a}^{b}|K(x, \xi)|^{2} d \xi \leqq B^{2}
$$

Hence,

$$
\left|\sum_{j=l}^{m}\left(f, \varphi_{j}\right) \lambda_{j} \varphi_{j}(x)\right| \leqq B\left[\sum_{j=l}^{m}\left|\left(f, \varphi_{j}\right)\right|^{2}\right]^{1 / 2}
$$

for all $x \in[a, b]$, from which we see that the sequence of the partial sums $S_{l}(x)$ of the series in (32) is uniformly Cauchy on $[a, b]$. Each $\varphi_{j}$ is continuous by Lemma 3 , so that $S_{l} \in C([a, b])$ for each $l$. Hence the limit of the partial sums $S_{l}(x)$ is also continuous on $[a, b]$. But $\left\{S_{l}\right\}$ converges to $\hat{K} f$ in $L^{2}([a, b])$ and $\hat{K} f \in C([a, b])$ by (31). Hence the function to which $\left\{S_{l}(x)\right\}$ converges uniformly must be $\hat{K} f$, which completes the proof.

As an immediate consequence of Theorem 1, Theorem 3, and Lemma 3, we have the following theorem: 
THEOREM 4. Let $\left\{\eta_{1}, \cdots, \eta_{n}\right\}$ be a linearly independent set of boundary functionals for $L$ and let $L$ be self-djoint on $M=\{f \epsilon$ $\left.C^{n}([a, b]) \mid \eta_{j}(f)=0,1 \leqq j \leqq n\right\}$. Let $\left\{\phi_{j}\right\}$ be the complete system of orthonormal eigenfunctions of $L$ and $\mu_{j}$ be the eigenvalue corresponding to $\varphi_{j}$. Then for every $f \in C([a, b])$ the solution of the generalized boundary value problem

$$
\begin{aligned}
L y & =f \\
\eta_{j}(y) & =0, \quad 1 \leqq j \leqq n
\end{aligned}
$$

exists and can be represented by the series

$$
\sum_{j=1}^{\infty} \mu_{j}^{-1}\left(f, \varphi_{j}\right) \varphi_{j}
$$

the series converging to the solution uniformly on $[a, b]$.

In order to facilitate the statements of the following two theorems, we state conditions and definitions used in the theorems.

(i) $\left\{\eta_{1}, \cdots, \eta_{l}\right\}, 1 \leqq l \leqq n$, is a set of boundary functionals for $L$, in which $\left\{\eta_{1}, \cdots, \eta_{r}\right\}$ is linearly independent in the sense of Definition 2.

(ii) $M_{0}=\left\{f \in C^{n}([a, b]) \mid \eta_{j}(f)=0,1 \leqq j \leqq l\right\}$.

(iii) $L$ is self-adjoint on $M_{0}$.

(iv) $N=M_{0} \cap S ; \operatorname{dim} N=p=n-r ; q=l-r$.

(v) $\left\{\psi_{1}, \cdots, \psi_{p}\right\}$ is an orthogonal basis for $N$.

(vi) $\zeta_{j}(f)=\left(f, \psi_{j}\right), f \in L^{2}([a, b]), 1 \leqq j \leqq p$. Then each $\zeta_{j}$ is a boundary functional for $L$ and the set $\left\{\eta_{1}, \cdots, \eta_{r}, \zeta_{1}, \cdots, \zeta_{p}\right\}$ is linearly independent in the sense of Definition 2.

(vii) $M_{1}=\left\{f \in C^{n}([a, b]) \mid \begin{array}{l}\eta_{j}(f)=0,1 \leqq j \leqq r \\ \zeta_{k}(f)=0,1 \leqq k \leqq p\end{array}\right\}$. Then by Theorem 1 , there exists a compact operator $K$ such that $L K f=f$ and $K f \in M_{1}$ for all $f \in C([a, b])$.

(viii) $\left\{\Phi_{j}\right\}$ is the complete system of (orthonormal) eigenfunctions of $L$ in $M_{0} \cap M_{1}$ and $\mu_{j}$ is the eigenvalue corresponding to $\varphi_{j}$, that is, $\varphi_{j} \in M_{0} \cap M_{1}$ and $L \varphi_{j}=\mu_{j} \varphi_{j}$.

Theorem 5. For every $f \in C([a, b])$ we have

$$
K f=\sum_{j=1}^{\infty} \mu_{j}^{-1}\left(f, \varphi_{j}\right) \varphi_{j}+\sum_{j=1}^{q}\left(f, \psi_{j}\right) K \psi_{j}
$$

and the series (together with the second sum) converges to $K f$ uniformly on $[a, b]$.

Proof. Since $L$ is self-adjoint on $M_{0} \cap M_{1}, K$ is self-adjoint on $L\left(M_{0} \cap M_{1}\right)$, the image of $M_{0} \cap M_{1}$ under $L$. We want to show that 
$L\left(M_{0} \cap M_{1}\right)$ is invariant under $K$. We first show that $K g \in M_{0} \cap M_{1}$ for every $g \in C([a, b]) \cap N^{\perp}$. To this end we show that every $f \in C([a, b])$ can be expressed in the form

$$
g+\sum_{j=1}^{q}\left(f, \psi_{j}\right) \psi_{j}
$$

with $g \in L\left(M_{0} \cap M_{1}\right)$.

Let $e_{k}=K \psi_{k}, 1 \leqq k \leqq q$. We claim that the determinant of the $q \times q$ matrix $\left[\eta_{r+i}\left(e_{j}\right)\right]$ is not zero. Suppose the contrary. Then there exist $\alpha_{j} \in C$, not all zero, such that

$$
\sum_{j=1}^{q} \eta_{r+i}\left(e_{j}\right) \alpha_{j}=0, \quad 1 \leqq i \leqq q,
$$

or

$$
\eta_{r+i}\left(\sum_{j=1}^{q} \alpha_{j} e_{j}\right)=0, \quad 1 \leqq i \leqq q .
$$

Since $\sum_{j=1}^{q} \alpha_{j} e_{j} \in M_{1}$, it follows $\eta_{k}\left(\sum_{j=1}^{q} \alpha_{j} e_{j}\right)=0,1 \leqq k \leqq l$, that is, $\sum_{j=1}^{q} \alpha_{j} e_{j} \in M_{0}$. Hence, $\left(L\left(\sum_{j=1}^{q} \alpha_{j} e_{j}\right), \psi\right)=0$ for all $\psi \in N$ since $L$ is self-adjoint on $M_{0}$ and $N \subset M_{0}$. Since $L\left(\sum_{i=1}^{q} \alpha_{i} e_{i}\right)=\sum_{j=1}^{q} \alpha_{j} \psi_{j} \in N$, it follows that $\sum_{j=1}^{q} \alpha_{j} \psi_{j}=0$, which implies $\alpha_{j}=0,1 \leqq j \leqq q$, contradicting the choice of $\alpha_{j}^{\prime}$ 's. Hence, $\operatorname{det}\left[\eta_{r+i}\left(e_{j}\right)\right] \neq 0$. Let $f \in C([a, b])$. Then $K f \in M_{1}$, and since $\operatorname{det}\left[\eta_{r+i}\left(e_{j}\right)\right] \neq 0$, there exists a unique set $\left\{\beta_{j}\right\}_{1}^{q}, \beta_{j} \in C$, such that

$$
\sum_{j=1}^{q} \eta_{r+i}\left(e_{j}\right) \beta_{j}=\eta_{r+i}(K f), \quad 1 \leqq i \leqq q,
$$

or

$$
\eta_{r+i}\left(K f-\sum_{j=1}^{q} \beta_{j} e_{j}\right)=0, \quad 1 \leqq i \leqq q,
$$

from which we have

$$
K f-\sum_{j=1}^{q} \beta_{j} e_{j} \in M_{0} \cap M_{1} .
$$

Let $g=f-\sum_{j=1}^{q} \beta_{j} \psi_{j}$. Then $K g \in M_{0} \cap M_{1}$ and we have

$$
f=g+\sum_{j=1}^{q} \beta_{j} \psi_{j} .
$$

Since $g=L(K g) \in L\left(M_{0} \cap M_{1}\right)$ and $L$ is self-adjoint on $M_{0}$, we have $(g, \psi)=0$ for all $\psi \in N$. Hence $\left(f, \psi_{k}\right)=\left(g, \psi_{k}\right)+\sum_{j=1}^{q} \beta_{j}\left(\psi_{j}, \psi_{k}\right)=\beta_{k}$, $1 \leqq k \leqq q$. Thus, for every $f \in C([a, b])$

$$
f=g+\sum_{j=1}^{q}\left(f, \psi_{j}\right) \psi_{j}
$$


with $K g \in M_{0} \cap M_{1}$. It follows immediately from (33) that

$$
K f \in M_{0} \cap M_{1} \text { for all } f \in C([a, b]) \cap N^{-} \text {. }
$$

To prove the invariance of $L\left(M_{0} \cap M_{1}\right)$ under $K$, let $g \in L\left(M_{0} \cap M_{1}\right)$. Then $K g \in M_{0} \cap M_{1}$, and since $M_{1} \subset N^{-}$, we have $K g \in N^{\perp}$. Moreover, $K g \in C([a, b])$. Hence from (34), $K(K g) \in M_{0} \cap M_{1}$ or $K g \in L\left(M_{0} \cap M_{1}\right)$. Thus $L\left(M_{0} \cap M_{1}\right)$ is invariant under $K$.

We let:

$K_{0}$ be the restriction of $K$ to $L\left(M_{0} \cap M_{1}\right)$,

$H_{0}$ be the closure of $L\left(M_{0} \cap M_{1}\right)$ in $L^{2}([a, b])$,

$\hat{K}_{0}$ be the continuous extension of $K_{0}$ to $H_{0}$.

Then $\hat{K}_{0}$ is a compact self-adjoint operator on the Hilbert space $H_{0}$. We next show that if $\varphi \in H_{0}$ and $\lambda \neq 0$ such that $\hat{K}_{0} \varphi=\lambda \varphi_{0}$, then $\varphi \in L\left(M_{0} \cap M_{1}\right)$.

Since $K$ is representable as an integral operator by Theorem 1 , $\hat{K}_{0}$ is representable as an integral operator and consequently just as in the case of Theorem 1 we can show that

$$
\hat{K}_{0} f \in C([a, b]) \text { for all } f \in H_{0},
$$

so that $\varphi=\lambda^{-1} K_{0} \varphi \in C([a, b])$. Hence by (33) we can write $\varphi=g+$ $\sum_{j=1}^{q}\left(\varphi, \psi_{j}\right) \psi_{j}$ with $g \in L\left(M_{0} \cap M_{1}\right)$. But $(f, \psi)=0$ for all $f \in L\left(M_{0} \cap M_{1}\right)$ and for all $\psi \in N$. It follows from this that $(f, \psi)=0$ for all $f \in H_{0}$ and for all $\psi \in N$. Hence $\left(\varphi, \psi_{j}\right)=0,1 \leqq j \leqq q$, so that $\varphi=g \in$ $L\left(M_{0} \cap M_{1}\right)$. Hence $K \varphi=K_{0} \varphi=\hat{K}_{0} \varphi=\lambda \varphi$ and so $L \varphi=\lambda^{-1} \varphi$. It follows from this that $\left\{\varphi_{j}\right\}$ is also the complete system of (orthonormal) eigenfunctions of $K_{0}$ corresponding to the nonzero eigenvalues of $K_{0}$. Hence by the same argument as in the proof of Theorem 3, for every $f \in H_{0}$

$$
\hat{K}_{0} f=\sum_{j=1}^{\infty}\left(\hat{K}_{0} f, \varphi_{j}\right) \varphi_{j}=\sum_{j=1}^{\infty} \mu_{j}^{-1}\left(f, \varphi_{j}\right) \varphi_{j}
$$

the series converging to $\hat{K}_{0} f$ uniformly on $[a, b]$. Now let $f \in C([a, b])$ and

$$
g=f-\sum_{j=1}^{q}\left(f, \psi_{j}\right) \psi_{j} .
$$

Then from (33) $g \in L\left(M_{0} \cap M_{1}\right)$, so that

$$
\begin{aligned}
K f & =K_{0} g+\sum_{j=1}^{q}\left(f, \psi_{j}\right) K \psi_{j} \\
& =\sum_{j=1}^{\infty} \mu_{j}^{-1}\left(g, \varphi_{j}\right) \varphi_{j}+\sum_{j=1}^{q}\left(f, \psi_{j}\right) K \dot{\psi}_{j} \\
& =\sum_{j=1}^{\infty} \mu_{j}^{-1}\left(f, \varphi_{j}\right) \varphi_{j}+\sum_{j=1}^{q}\left(f, \psi_{j}\right) K \psi_{j}
\end{aligned}
$$


since $\left(\psi_{k}, \varphi_{j}\right)=0$ for all $k$ and $j$, and the series (together with the second sum) converges to $K f$ uniformly on $[a, b]$.

THEOREM 6. Under the conditions (i)-(viii), for every $f \in$ $C([a, b]) \cap N^{\perp}$, the generalized homogeneous boundary value problem

$$
\begin{aligned}
L y & =f \\
\eta_{j}(y) & =0, \quad 1 \leqq j \leqq l
\end{aligned}
$$

has a unique solution in $M_{0}$ and the solution can be represented by the series

$$
\sum_{j=1}^{\infty} \mu_{j}^{-1}\left(f, \varphi_{j}\right) \varphi_{j}
$$

the series converging to the solution uniformly on $[a, b]$.

\section{REFERENCES}

1. J. W. Calkin, Abstract symmetric boundary conditions, Trans. Amer. Math. Soc., 45 (1939), 369-442.

2. N. Dunford and J. T. Schwartz, Linear operators II: spectral theory, Pure and Appl. Math., 7, Interscience, New York, 1963.

3. M. A. Naimark, Linear Differential Operators, I, Ungar, New York, 1967.

4. K. Yoshida, Lectures on differential and integral equations, Pure and Appl. Math., 10, Interscience, New York, 1960.

Received May 8, 1973 and in revised form October 29, 1973.

UNIVERSITY OF HAWAII, HONOLULU 


\section{PACIFIC JOURNAL OF MATHEMATICS}

\section{EDITORS}

RICHARD ARENS (Managing Editor)

University of California

Los Angeles, California 90024

R. A. Beaumont

University of Washington

Seattle, Washington 98105
J. DugundJI

Department of Mathematics University of Southern California Los Angeles, California 90007

D. Gilbarg AND J. Milgram Stanford University

Stanford, California 94305

\section{ASSOCIATE EDITORS}
E. F. BECKENBACH
B. H. NeUmanN
F. WOLF
K. YOSHIDA

\section{SUPPORTING INSTITUTIONS}

UNIVERSITY OF BRITISH COLUMBIA CALIFORNIA INSTITUTE OF TECHNOLOGY

UNIVERSITY OF CALIFORNIA

MONTANA STATE UNIVERSITY

UNIVERSITY OF NEVADA

NEW MEXICO STATE UNIVERSITY

OREGON STATE UNIVERSITY

UNIVERSITY OF OREGON

OSAKA UNIVERSITY
UNIVERSITY OF SOUTHERN CALIFOF

STANFORD UNIVERSITY

UNIVERSITY OF TOKYO

UNIVERSITY OF UTAH

WASHINGTON STATE UNIVERSITY UNIVERSITY OF WASHINGTON

AMERICAN MATHEMATICAL SOCIET NAVAL WEAPONS CENTER 


\section{Pacific Journal of Mathematics}

\section{Vol. 54, No. 1 \\ May, 1974}

Ralph K Amayo, Engel Lie rings with chain conditions ..................

Bernd Anger and Jörn Lembcke, Hahn-Banach type theorems for hypolinear

functionals on preordered topological vector spaces ..................

Gregory Frank Bachelis and Samuel Ebenstein, On $\Lambda(p)$ sets ................

Harvey Isaac Blau, Indecomposable modules for direct products of finite

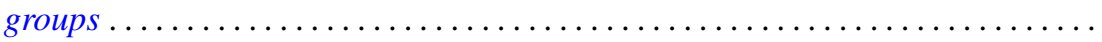

Larry Eugene Bobisud and James Calvert, Singular perturbation of a

time-dependent Cauchy problem in a Hilbert space ................

Walter D. Burgess and Robert Raphael, Abian's order relation and orthogonal

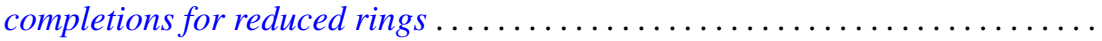

James Diederich, Representation of superharmonic functions mean continuous at

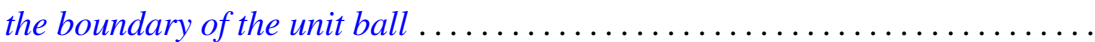

Aad Dijksma and Hendrik S. V. de Snoo, Self-adjoint extensions of symmetric

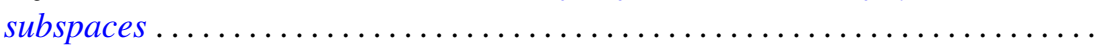

Gustave Adam Efroymson, A Nullstellensatz for Nash rings . . . . . . . . . . . . .

John D. Elwin and Donald R. Short, Branched immersions onto compact orientable surfaces . . . . . . . . . . . . . . . . . . . . . . . . .

John Douglas Faires, Comparison of the states of closed linear

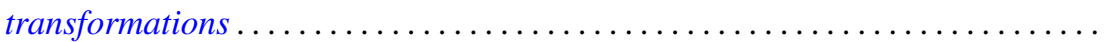

Joe Wayne Fisher and Robert L. Snider, On the von Neumann regularity of rings with regular prime factor rings .

Franklin Takashi Iha, A unified approach to boundary value problems on compact intervals

Palaniappan L. Kannappan and Che Tat $\mathrm{Ng}$, On functional equations connected with directed divergence, inaccuracy and generalized directed divergence

Samir A. Khabbaz and Elias Hanna Toubassi, The module structure of Ext $(F, T)$ over the endomorphism ring of $T$...

Garo K. Kiremidjian, On deformations of complex compact manifolds with boundary.

Dimitri Koutroufiotis, Mappings by parallel normals preserving principal

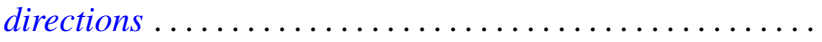

W. K. Nicholson, Semiperfect rings with abelian adjoint group

Norman R. Reilly, Extension of congruences and homomorphisms to translational hulls

Sadahiro Saeki, Symmetric maximal ideals in $M(G)$

Brian Kirkwood Schmidt, On the homotopy invariance of certain functors ...

H. J. Shyr and T. M. Viswanathan, On the radicals of lattice-ordered rings ...

Indranand Sinha, Certain representations of infinite group algebras ...

David Smallen, The group of self-equivalences of certain complexes ...

Kalathoor Varadarajan, On a certain problem of realization in homotopy

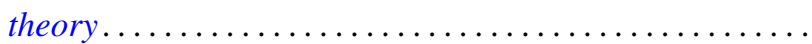

